\title{
Comportement hydrogéochimique et contamination des eaux des aquifères fissurés du socle précambrien en milieu semi-aride (Sud-Ouest du Niger)
}

\author{
Maman Sani ABDOU BABAYE ${ }^{1,2^{*}}$, Issoufou SANDAO², Mahaman Bachir SALEY ${ }^{3}$, \\ Ibrahim WAGANI ${ }^{1}$ et Boureima OUSMANE ${ }^{2}$ \\ ${ }^{1}$ Université Dan Dicko Dankoulodo Faculté des Sciences et Techniques, UMR SERMUG, Département de \\ Géologie, BP:465, Marad (Niger). \\ ${ }^{2}$ Université Abdou Moumouni, Faculté des Sciences et Techniques, Département de Géologie, BP:10662 \\ Niamey (Niger). \\ ${ }^{3}$ Université Félix Houphouët Boigny d'Abidjan - Cocody, UFR SRTM, CURAT - 22 BP 801 Abidjan 22 (Côte \\ d'Ivoire) \\ *Auteur correspondant ; E-mail : babaye.sani@gmail.com
}

\section{REMERCIEMENTS}

Cette étude a été réalisée grâce aux soutiens financiers de la CTB et de l'IFS. Nous leur exprimons notre profonde gratitude.

\section{RESUME}

Située dans la partie Sud-Ouest du Niger, la zone d'étude appartient à la région de socle précambrien du Liptako. Dans cette zone, la faible couverture en eau est accentuée par le problème de la qualité car certains forages enregistrent une teneur importante en nitrates. L'objectif de cette étude est d'améliorer les connaissances sur la qualité des eaux de socle, et déterminer les sources et les facteurs qui contrôlent leur minéralisation. Ce travail a été réalisé à partir des résultats d'analyses physico-chimiques de cinquante échantillons d'eau prélevés au niveau des puits peu profonds et des forages. Dans cette étude, une méthodologie combinant les approches hydrochimiques classiques et l'analyse en composante principale est utilisée. L'étude du chimisme des eaux souterraines révèle trois types des faciès dont le plus dominant est le type bicarbonaté calco-magnésien (70\%). Environ $40 \%$ d'ouvrages ont des teneurs en nitrates comprises entre 50 et $690 \mathrm{mg} / \mathrm{l}$ (normes OMS $50 \mathrm{mg} / \mathrm{l}$ ). L'origine de la minéralisation des eaux des aquifères de socle est due à l'hydrolyse des minéraux silicatés, mais aussi aux apports superficiels.

(C) 2016 International Formulae Group. All rights reserved.

Mots clés : aquifères fissurés, qualité des eaux, contamination, hydrogéochimie, Niger.

\section{Hydrogeochemical behavior and contamination of waters cracked aquifer of the precambrian basement in semi-arid areas (southwestern Niger)}

\begin{abstract}
Located in the southwest part of the Niger, the study area belongs to the precambrian basement of Liptako. In this area, the low water coverage is accentuated by the problem of water quality as in some
\end{abstract}


boreholes it is recorded a high content of nitrates. The objective of this study is to improve the knowledge basis on the water quality and to determine the sources and factors that control their mineralization. In this study, a methodology combining classical hydrochemical approaches and principal component analysis is used. The groundwater chemistry of reveals three types of facies. The most dominant is the type calc-magnesium bicarbonate with about $70 \%$. Approximately, $40 \%$ of boreholes have a nitrate content ranging between 50 and $690 \mathrm{mg} / \mathrm{l}$ while the WHO standards is of $50 \mathrm{mg} / \mathrm{l}$. The mineralization origin of the bedrock aquifers of water is due to hydrolysis of the silicate minerals, but also to superficial contributions.

(c) 2016 International Formulae Group. All rights reserved.

Keywords: fissured aquifers, water quality, contamination, Hydrogeochemistry, Niger.

\section{INTRODUCTION}

Depuis la fin des années 1960, les zones sahéliens connaissent de profonds changements climatiques caractérisés par une baisse considérable des précipitations (Taylor et al., 2002). Dans ces zones à l'environnement sensible, les sécheresses répétitives ont eu pour conséquences la dégradation des états de surface, et le changement durable du bilan hydrologique (Leblanc et al., 2008 ; Favreau et al., 2009).

Les ressources en eau souterraine des zones sahéliennes, et plus particulièrement celles des régions de socle du Sud-Ouest du Niger, connaissent de plus en plus une dégradation qualitative et quantitative, du fait des actions climatiques et anthropiques. Si en zone tropicale humide, la nature offre plusieurs alternatives en matière de production d'eau potable de la population, en zone semiaride de socle, les aquifères fissurés restent les seuls points de recours pendant les longues saisons sèches ( $2 / 3$ de l'année).

Vu l'importance remarquable de ces ressources pour améliorer le taux de desserte en eau de la population, le suivi de la qualité physico-chimique de ces eaux devient un impératif surtout que certains forages enregistrent des teneurs élevées en nitrate (Elbaz-Poulichet, 2002; Yaméogo, 2008). L'étude de la qualité physico-chimique des eaux devient impérative dans le schéma régional de gestions rationnelles des ressources en eau souterraine de socle. Cette étude a pour objectif de définir les caractéristiques physico-chimiques des eaux de socle, et de déterminer les sources et les facteurs qui contrôlent leur minéralisation.

\section{MATERIEL ET METHODES}

\section{Situation de la zone d'étude}

La zone d'étude se situe dans la partie Nord-Ouest du Liptako nigérien entre les longitudes $0^{\circ} 30^{\prime}$ 'Ouest et $0^{\circ} 50^{\prime}$ 'Est et les latitudes $13^{\circ} 45^{\prime}$ et $14^{\circ} 20^{\prime}$ Nord. Elle occupe la partie centrale de Dargol qui est un affluent rive droite du fleuve Niger (Figure 1).

Le climat de la zone est du type sahélien caractérisé par l'alternance de deux saisons très contrastées: une saison sèche d'octobre à mai et une saison de pluies allant de juin à octobre. La pluviométrie moyenne annuelle calculée à la station pluviométrique de Téra sur la période 1999 - 2008 est de 409 $\mathrm{mm}$. Au cours de la même période, les valeurs moyennes mensuelles de la température varient entre $15{ }^{\circ} \mathrm{C}$ à $43{ }^{\circ} \mathrm{C}$, pour une moyenne annuelle de $29{ }^{\circ} \mathrm{C}$. Les températures les plus basses sont observées en juillet-août (saison hivernale) et décembre-janvier (saison "froide"), tandis que les fortes valeurs s'observent en avril-mai (saison sèche) et octobre-novembre. Pendant la saison sèche, on observe généralement des pics pouvant atteindre $43{ }^{\circ} \mathrm{C}$ à l'ombre. Les taux de l'humidité relative de l'air montrent une variation annuelle de 18 à $90 \%$. L'évapotranspiration potentielle annuelle est supérieure à $2000 \mathrm{~mm}$ dépassant quatre fois la hauteur pluviométrique annuelle (Abdou Babaye, 2012). 
Le réseau hydrographique comprend un cours d'eau temporaire (le Dargol) dont le plus important des affluents est le Tilim. À cette rivière, s'ajoute un chapelet de mares semi-permanentes et des retenues artificielles d'eau parmi lesquelles, seul le barrage de Téra est permanent.

\section{Contexte géologique et hydrogéologique}

Le substratum de la zone est constitué des roches précambriennes (Figure 1) réparties entre les formations de la ceinture métamorphique (pyroxénites, amphibolites, épidotites, chloritoschistes, métabasaltes, métagabbros, grauwackes, micaschistes, quartzitoschistes, tufs et brèches rhyolitiques) et celles du pluton granitoïdique (granites, granodiorites et diorites) renfermant par endroit des reliques archéennes (pegmatites, leptynites) (Machens, 1973; Abbou et al., 1998; Soumaila, 2000). Les formations de socle sont surmontées d'une couverture superficielle formée d'altérite (5 à $50 \mathrm{~m}$ d'épaisseur), des alluvions et des colluvions. Dans cette zone, l'essentiel des ressources en eau souterraine est contenu dans les aquifères fissurés de socle précambrien dont l'hydrogéologie est mal connue.

En milieu cristallin et cristallophyllien, les zones aquifères correspondent aux niveaux superficiels altérés (altérites et alluvions) et profonds de socle (fissures et/ou fractures), qui sont dans bien des cas superposés et forment un même système aquifère (Dewandel et al., 2006). L'aquifère supérieur est formé par des matériaux semi-perméables au rôle capacitif, suivi d'un horizon fissuré (zone intermédiaire décomprimée), à perméabilité élevée formant le deuxième niveau aquifère. Ce type de fonctionnement a été indiqué comme adapté à celui relatif au socle précambrien de la zone d'étude (Ousmane, 1988; Girard et al., 1997). Le troisième niveau aquifère se trouve dans le substratum sain affecté par des fractures d'origine tectonique (failles). Cet aquifère profond de socle fissuré et fracturé est en général confiné, tout en étant par endroit drainant par rapport aux horizons supérieurs. Les zones les plus fracturées contribuent donc à sa fonction essentiellement conductrice. Le degré de confinement est fonction $\mathrm{du}$ pourcentage des matériaux argileux. Dans le domaine schisteux et de roches vertes, la couche altérée est très épaisse (15 à $40 \mathrm{~m}$ ) et à dominance argileuse, contrairement au terrain granitique (Ousmane, 1988).

\section{Méthodologie \\ Echantillonnage et analyses chimiques}

La présente étude est basée sur cinquante échantillons d'eau prélevés au niveau des puits peu profonds et des forages. Les prélèvements ont été réalisés au cours des périodes de hautes (45 échantillons en octobre à décembre 2009) et de basses eaux (6 échantillons en avril-mai 2010) et les échantillons sont distinctement étiquetés selon la nappe et la date d'échantillonnage. Les points d'échantillonnage sont choisis de manière à représenter les différents ensembles géologiques rencontrés dans la zone d'étude. La quasi-totalité des mesures ont porté sur les aquifères fissurés de socle. Néanmoins, une analyse a concerné la nappe superficielle d'altérites. Certains points ont fait l'objet de deux ou trois prélèvements. Au cours de chaque campagne d'échantillonnage, les paramètres physico-chimiques in situ $(\mathrm{pH}$, température, conductivité électrique) sont mesurés in situ. Les mesures de ces paramètres et les prélèvements ont été effectués sur les ouvrages en fonctionnement après au moins 30 minutes de pompage (forages) ou de puisage (puits) pour obtenir une eau plus représentative de la nappe. Les analyses chimiques réalisées par méthodes usuelles présentent les balances ioniques variant autour de $\pm 3 \%$. Les dosages des éléments chimiques sont réalisés sur une électrophorèse capillaire. Les cations $\left(\mathrm{Ca}^{2+}\right.$, $\mathrm{Mg}^{2+}, \mathrm{Na}^{+}$et $\mathrm{K}^{+}$) et la silice sont déterminés 
par absorption atomique de flamme. L'analyse des nitrates $\left(\mathrm{NO}_{3}{ }^{-}\right)$et des chlorures $\left(\mathrm{Cl}^{-}\right)$a été faite par titration potentiométrique directe, alors que les ions carbonatés $\left(\mathrm{CO}_{3}{ }^{2-}\right)$ et bicarbonatés $\left(\mathrm{HCO}_{3}{ }^{-}\right)$sont obtenus par le calcul après la détermination du $\mathrm{pH}$ et du titre alcalimétrique complet (TAC).

\section{Traitements des données}

Les résultats analytiques sont traités à l'aide des diagrammes et par les techniques statistiques.

La classification hydrogéochimique des eaux a été réalisée grâce au diagramme de Piper. Ce dernier est fréquemment utilisé pour la typologie des eaux. Les éléments chimiques considérés pour ce traitement concernent les cations $\mathrm{Ca}^{2+}, \mathrm{Mg}^{2+},\left(\mathrm{Na}^{+}+\mathrm{K}^{+}\right)$et les anions $\mathrm{HCO}^{3-},\left(\mathrm{Cl}^{-}+\mathrm{NO}^{3-}\right)$ et $\mathrm{SO}_{4}{ }^{2-}$. Ainsi, les faciès géochimiques des eaux sont obtenus en fonction de la prédominance des cations et des anions dans la solution.

Pour mieux apprécier l'influence des ions sur la minéralisation des eaux, deux approches statistiques ont été utilisées. La première consiste à l'analyse bivariable afin de mettre en évidence les corrélations des ions pris deux à deux d'une part, et leurs apports sur l'évolution hydrogéochimique des eaux. La deuxième analyse est basée sur l'exploitation des résultats obtenus à partir la technique d'Analyse en Composantes Principales (ACP). L'ACP centrée réduite a été réalisée avec 12 paramètres (conductivité électrique (CE), la minéralisation totale (MT), le $\mathrm{pH}$, la pression partielle en $\mathrm{CO}_{2}\left(\mathrm{pCO}_{2}\right)$, $\mathrm{Ca}^{2+}, \mathrm{Mg}^{2+}, \mathrm{Na}^{+}, \mathrm{K}^{+}, \mathrm{HCO}^{3-}, \mathrm{SO}_{4}{ }^{2-}, \mathrm{Cl}^{-}, \mathrm{NO}_{3}{ }^{-}$) à l'aide du logiciel STATISTICA 6.1. L'intérêt de cette technique est qu'elle permet de résumer à quelques axes factoriels le maximum d'informations contenues dans la masse de données. La projection des individus sur un plan factoriel offre l'avantage d'appréhender la similitude ou l'opposition entre les caractéristiques des individus et les sources de leur variabilité. Le nuage des points formés par le regroupement de certains ions impose une étude bivariable afin d'apprécier l'apport de chaque ion dans la minéralisation des eaux.

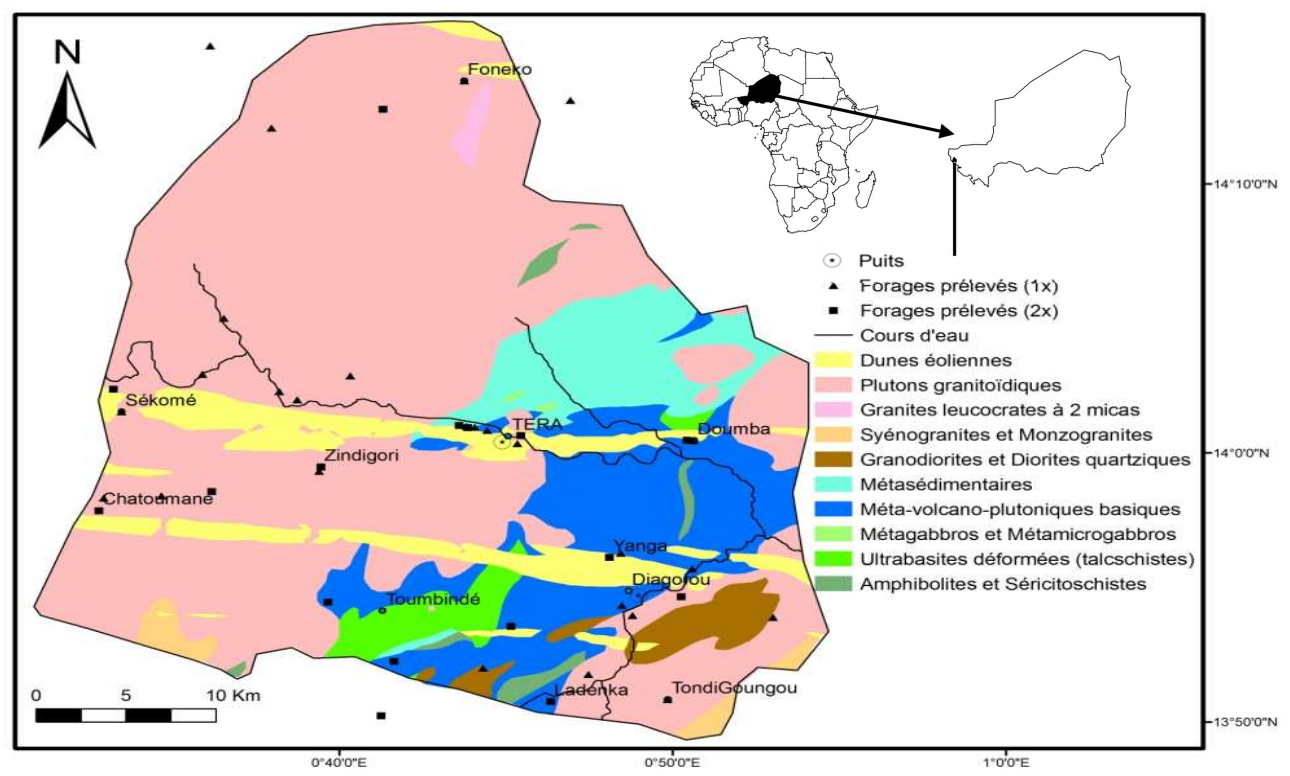

Figure 1 : Localisation de la zone d'étude, contexte géologique et distribution des points d'eau échantillonnés. $(1 \times)$ : prélevés une fois. 


\section{RESULTATS}

Analyses physico-chimiques

Les résultats des analyses physicochimiques (Tableau 1) révèlent que les eaux souterraines sont légèrement acides à neutres, avec un pH qui varie de 6,30 à 7,30. Elles se caractérisent par une minéralisation très variable (266 à $1731 \mu \mathrm{s} / \mathrm{cm})$ avec une moyenne de $617 \mu \mathrm{s} / \mathrm{cm}$. Cette grande variation montre l'hétérogénéité notable dans la distribution de la charge minérale des eaux souterraines. Elle montre aussi que la minéralisation des eaux souterraine en zone de socle est en générale plus élevée au niveau des aquifères des roches vertes (schiste) que sur le terrain granitique. Cela est lié au confinement de ces aquifères par d'épaisse couche argileuse. Les valeurs de la température de l'eau varie de 29 à $34{ }^{\circ} \mathrm{C}$ dans l'ensemble des échantillons d'eaux souterraines. Pour ce qui est des anions, les bicarbonates, avec une valeur moyenne de $258 \mathrm{mg} / \mathrm{l}$, dominent largement tous les anions. En ce qui concerne les ions $\mathrm{Cl}^{-}, \mathrm{SO}_{4}{ }^{2-}$ et $\mathrm{NO}_{3}{ }^{-}$, leurs valeurs maximales sont respectivement 106,81 mg/l, $294,95 \mathrm{mg} / \mathrm{l}$ et 0,3 à $690,16 \mathrm{mg} / \mathrm{l}$. La teneur en sulfates est acceptable selon les normes OMS (400 mg/l), mais pour les nitrates, 40\% d'ouvrages enregistrent des teneurs dépassant les normes de potabilité acceptées par l'OMS $(\leq 50 \mathrm{mg} / \mathrm{l})$. Cette pollution nitratée s'observe au niveau de toutes les catégories des eaux souterraines (puits et forages).

Les valeurs moyennes des cations des eaux analysées présentent l'ordre d'abondance suivant: $\mathrm{Ca}^{2+}>\mathrm{Na}^{+}>\mathrm{Mg}^{2+}>\mathrm{K}^{+}$. Parfois, le sodium devient prépondérant dans les eaux des ouvrages proches des zones potentielles de recharge (koris, mares). Aussi, la teneur en sodium se voit dans certains cas dépasser par le magnésium dû à l'importance de terrain schisteux dans la zone et aux intrusions doléritiques dans les massifs granitoïdiques.

La classification chimique des eaux révèle une abondance des eaux bicarbonatées calco-magnésiennes (70\%) suivies des eaux chloruro-sulfatées calciques $(25 \%$ et des eaux bicarbonatées sodiques (5\%) (Figure 2). La signature chloruro-sulfatée calcique des eaux marque l'évolution des anions du pôle bicarbonaté vers le pôle chloruré-nitraté. Cette évolution est principalement due à une augmentation des nitrates, et de manière secondaire par l'apport des sulfates dans l'évolution géochimique vers un troisième faciès.

\section{Analyses statistiques}

Le Tableau 2 fait ressortir les corrélations existantes entre les 12 variables prises deux à deux. Ainsi, le $\mathrm{pH}$ est bien corrélé aux $\mathrm{HCO}_{3}{ }^{-}(0,73)$ mais anté-corrélé au $\mathrm{pCO}_{2}(-0,83)$; la conductivité électrique est fortement corrélée avec MT $(0,94), \mathrm{Ca}^{2+}$ $(0,91), \mathrm{NO}_{3}^{-}(0,85), \mathrm{Cl}^{-}(0,80), \mathrm{Mg}^{2+}(0,78)$ et assez bien corrélée au $\mathrm{Na}^{+}(0,55)$; le $\mathrm{HCO}_{3}{ }^{-}$ est corrélé au $\mathrm{Na}^{+}(0,55)$; le $\mathrm{Ca}^{2+}$ est bien corrélé avec les $\mathrm{NO}_{3}{ }^{-}(0,89), \mathrm{Cl}^{-}(0,84), \mathrm{Mg}^{2+}$ $(0,71)$; le $\mathrm{Mg}^{2+}$ est corrélé avec les $\mathrm{NO}_{3}{ }^{-}$ $(0,62)$ et peu corrélé aux $\mathrm{SO}_{4}{ }^{2-}(0,49)$ et $\mathrm{HCO}_{3}{ }^{-}(0,43)$; et enfin, le $\mathrm{Na}^{+}$est corrélé aux $\mathrm{HCO}_{3}^{-}(0,53)$.

Les résultats de l'ACP centrée réduite (Figure 3) montrent que la variance exprimée par les axes factoriels $\mathrm{F}_{1}-\mathrm{F}_{2}$ est assez significative (près de 70\%) pour observer le comportement global des échantillons (Tableau 3). Néanmoins, nous avons aussi considéré l'axe $F_{3}$ pour pouvoir mettre en évidence les tendances non exprimée et avoir des projections de bonne qualité (> 70\%). Les trois premiers axes factoriels $\left(\mathrm{F}_{1}, \mathrm{~F}_{2}, \mathrm{~F}_{3}\right)$ expriment près de $80 \%$ de la variance totale, ce qui est très significatif pour faire la classification et étudier les tendances et corrélations qui peuvent exister entre les paramètres afin de mieux en déduire les mécanismes d'acquisition de la minéralisation des eaux souterraines.

La projection des variables sur le plan factoriel $F_{1}-F_{2}$ (Figure 3) montre que le facteur $\mathrm{F}_{1}$ est le plus important. Il contrôle à lui seul environ $47 \%$ de l'inertie du nuage des points représentatifs des échantillons et regroupe au pôle négatif la majorité des variables en présence $\left(\mathrm{CE}, \mathrm{MT}, \mathrm{Ca}^{2+}, \mathrm{NO}_{3}{ }^{-}\right.$, $\mathrm{Cl}^{-}, \mathrm{Mg}^{2+}, \mathrm{Na}^{+}$et $\mathrm{SO}_{4}{ }^{2-}$ ). Le regroupement de ces variables autour de l'axe $\mathrm{F}_{1}$ montre que cet axe factoriel exprime les sources principales 
M. S. ABDOU BABAYE et al. / Int. J. Biol. Chem. Sci. 10(6): 2728-2743, 2016

Tableau 1 : Paramètres physico-chimiques (mg/l) des eaux.

\begin{tabular}{|c|c|c|c|c|c|c|c|c|c|c|c|c|c|c|c|}
\hline Nom & $\mathbf{N}^{\circ}$ & Aquifère & Long & Lat & $\mathrm{CE} 25^{\circ}$ & pH & $\mathbf{T}{ }^{\circ} \mathbf{C}$ & $\mathrm{Ca}^{2+}$ & $\mathrm{Mg}^{2+}$ & $\mathrm{Na}^{+}$ & $\mathbf{K}^{+}$ & $\mathrm{Cl}^{-}$ & $\mathrm{SO}_{4}{ }^{2-}$ & $\mathrm{NO}_{3}^{-}$ & $\mathrm{HCO}_{3}^{-}$ \\
\hline Diagourou & $\mathrm{F} 3$ & RV & 0,80809 & 13,90552 & 599 & 6,8 & 32,2 & 33,30 & 19,43 & 76,97 & 4,24 & 13,97 & 17,49 & 10,52 & 338,24 \\
\hline Diagourou jardin & $\mathrm{F} 2$ & RV & 0,8154 & 13,90968 & 376 & 6,8 & 30,4 & 31,84 & 16,59 & 31,17 & 2,12 & 5,88 & 9,88 & 12,58 & 218,99 \\
\hline Lambandou & F5 & RV & 0,83768 & 13,91097 & 814 & 6,4 & 31,5 & 18,72 & 10,51 & 19,11 & 2,97 & 4,05 & 3,69 & 2,04 & 146,81 \\
\hline Pate Kouara & F8 & RV & 0,81334 & 13,89929 & 385 & 6,6 & 32,9 & 24,49 & 12,27 & 47,75 & 3,01 & 11,23 & 9,75 & 1,40 & 229,60 \\
\hline Fanta Faouta & F10 & RV & 0,75254 & 13,89263 & 441 & 7,1 & 31,5 & 42,86 & 18,58 & 25,90 & 8,00 & 2,05 & 17,07 & 13,32 & 268,19 \\
\hline Tchantiga & F11 & RV & 0,69412 & 13,87092 & 573 & 6,9 & 32 & 48,24 & 36,83 & 33,41 & 3,73 & 3,41 & 9,19 & 6,30 & 390,18 \\
\hline Tampiana & F12 & RV & 0,68766 & 13,83725 & 582,8 & 6,9 & 32,2 & 68,73 & 20,68 & 31,32 & 7,20 & 2,67 & 46,14 & 50,66 & 289,28 \\
\hline Ladanka & F14 & RV & 0,77255 & 13,84599 & 583 & 7 & 32,8 & 39,46 & 40,54 & 46,28 & 2,32 & 6,18 & 18,88 & 12,41 & 377,14 \\
\hline Toumbinde & F15 & RV & 0,68814 & 13,90215 & 706,3 & 6,9 & 32,8 & 50,53 & 29,78 & 66,18 & 2,98 & 10,31 & 50,53 & 108,68 & 292,33 \\
\hline Kalbara & F16 & RV & 0,66111 & 13,90774 & 605 & 6,8 & 31,5 & 61,26 & 23,68 & 41,87 & 2,71 & 6,46 & 32,03 & 79,16 & 277,52 \\
\hline Doumba & F37 & RV & 0,8406 & 14,00804 & 1150,5 & 6,6 & 29,4 & 128,28 & 48,66 & 70,64 & 6,01 & 21,78 & 294,95 & 98,93 & 294,77 \\
\hline Doun Diyal & F38 & RV & 0,84386 & 14,00761 & 582 & 6,4 & 30,9 & 49,08 & 15,63 & 59,19 & 4,57 & 13,53 & 17,12 & 74,23 & 261,48 \\
\hline Warigountou & F41 & RV & 0,84308 & 13,92813 & 545,8 & 7,3 & 30,7 & 36,75 & 30,47 & 49,36 & 4,16 & 5,88 & 18,51 & 0,30 & 347,69 \\
\hline Yanga 1 & $\mathrm{~F} 44$ & RV & 0,80184 & 13,93535 & 968 & 6,9 & 32,8 & 95,68 & 39,16 & 51,04 & 4,77 & 11,50 & 33,78 & 281,35 & 275,81 \\
\hline Yanga 2 & $\mathrm{~F} 45$ & RV & 0,80736 & 13,93793 & 1123,3 & 7 & 32,3 & 111,38 & 45,18 & 71,58 & 4,74 & 23,43 & 178,06 & 231,10 & 250,94 \\
\hline Lata & F60 & RV & 0,73856 & 13,86664 & 630 & 6,8 & 31,9 & 48,47 & 27,74 & 47,78 & 3,53 & 12,05 & 31,65 & 32,61 & 300,87 \\
\hline Tondi Gougou & F6 & G & 0,8308 & 13,84719 & 985 & 6,7 & 32,1 & 79,68 & 26,86 & 55,53 & 1,04 & 27,99 & 27,42 & 187,70 & 258,56 \\
\hline Tondi Gougou II & F7 & $\mathrm{G}$ & 0,83446 & 13,84605 & 976 & 6,8 & 31,4 & 83,66 & 26,48 & 84,06 & 4,77 & 38,86 & 36,31 & 281,33 & 218,20 \\
\hline
\end{tabular}


M. S. ABDOU BABAYE et al. / Int. J. Biol. Chem. Sci. 10(6): 2728-2743, 2016

\begin{tabular}{|c|c|c|c|c|c|c|c|c|c|c|c|c|c|c|c|}
\hline Linlingo & F9 & G & 0,79126 & 13,86277 & 369 & 7 & 34 & 39,08 & 18,76 & 16,32 & 6,01 & 1,72 & 2,92 & 0,30 & 254,78 \\
\hline Foneko & F17 & G & 0,72917 & 14,23055 & 610,5 & 6,6 & 31,5 & 44,76 & 25,55 & 60,49 & 0,01 & 16,73 & 8,79 & 49,52 & 315,50 \\
\hline Kabangou & F18 & G & 0,73035 & 14,23062 & 1184,5 & 6,8 & 31,1 & 108,10 & 62,61 & 53,73 & 0,01 & 45,22 & 15,42 & 405,83 & 278,98 \\
\hline Fala I & $\mathrm{F} 20$ & G & 0,68854 & 14,21298 & 348,8 & 6,5 & 32,1 & 39,52 & 7,04 & 27,05 & 1,14 & 5,51 & 4,79 & 52,49 & 159,49 \\
\hline Zindigori & $\mathrm{F} 25$ & G & 0,65764 & 13,99133 & 350 & 6,5 & 31,2 & 26,29 & 9,36 & 34,53 & 1,62 & 10,74 & 11,94 & 57,45 & 128,39 \\
\hline Zindigori N & F26 & G & 0,65671 & 13,98874 & 665 & 6,7 & 31 & 55,07 & 22,49 & 69,47 & 3,47 & 14,52 & 12,58 & 32,74 & 372,44 \\
\hline Kababangou $\mathrm{H}$ & $\mathrm{F} 27$ & G & 0,60293 & 13,97608 & 338,5 & 6,6 & 31,5 & 35,34 & 11,03 & 27,80 & 2,13 & 4,88 & 4,99 & 2,66 & 218,01 \\
\hline Handaga & $\mathrm{F} 28$ & G & 0,57778 & 13,97346 & 641 & 6,8 & 31,1 & 50,03 & 25,46 & 58,46 & 5,59 & 11,23 & 8,98 & 37,95 & 356,59 \\
\hline Chatoumane & F30 & G & 0,54651 & 13,96425 & 784 & 6,9 & 32,3 & 60,86 & 23,09 & 80,33 & 4,39 & 16,19 & 19,63 & 129,63 & 329,77 \\
\hline $\begin{array}{l}\text { Chatoumane } \\
\text { cardia }\end{array}$ & $\mathrm{F} 31$ & G & 0,54885 & 13,97198 & 623 & 6,8 & 32,9 & 51,94 & 11,29 & 69,34 & 3,01 & 21,92 & 10,16 & 104,55 & 244,17 \\
\hline Sekomi Goria & F33 & G & 0,55387 & 14,03962 & 285,1 & 6,5 & 31,6 & 31,05 & 7,94 & 13,06 & 3,78 & 8,28 & 2,22 & 58,81 & 100,41 \\
\hline Tondi Karia & F36 & G & 0,60907 & 14,08355 & 227,7 & 6,3 & 30,5 & 18,52 & 10,17 & 17,67 & 1,43 & 2,66 & 2,24 & 0,30 & 149,98 \\
\hline Tera Mosquée & F50 & G & 0,75584 & 14,00587 & 1731 & 6,4 & 32,4 & 215,88 & 45,69 & 57,15 & 8,01 & 106,81 & 28,27 & 690,16 & 129,43 \\
\hline Sirfi Kouara & F49 & $\mathrm{G}$ & 0,76413 & 13,99906 & 541 & 6,7 & 29,1 & 55,66 & 10,57 & 53,03 & 3,44 & 8,44 & 40,67 & 15,82 & 269,47 \\
\hline Tera Campt & $\mathrm{F} 47$ & G & 0,751 & 14,01037 & 286,8 & 6,1 & 33 & 26,24 & 9,11 & 21,28 & 5,17 & 11,69 & 9,99 & 3,32 & 154,61 \\
\hline Tera Beria & F54 & G & 0,72677 & 14,0172 & 542 & 7 & 32,7 & 33,85 & 25,56 & 54,83 & 5,55 & 9,80 & 22,38 & 4,23 & 318,91 \\
\hline Tera Pont & $\mathrm{F} 48$ & G & 0,75746 & 14,0109 & 531 & 6,7 & 31,5 & 42,97 & 18,93 & 53,67 & 3,10 & 15,27 & 30,42 & 22,68 & 270,57 \\
\hline Tera T7 & F55 & G & 0,73444 & 14,01593 & 585 & 6,9 & 31,4 & 45,11 & 23,44 & 47,10 & 10,41 & 5,21 & 28,33 & 1,74 & 323,24 \\
\hline Tera T2 & F56 & G & 0,74077 & 14,01401 & 441,5 & 6,9 & 31 & 46,72 & 14,38 & 30,28 & 5,87 & 4,04 & 8,79 & 3,02 & 264,47 \\
\hline Fogou & F57 & G & 0,67227 & 14,04778 & 543 & 6,7 & 32,6 & 55,95 & 10,87 & 39,56 & 3,57 & 4,23 & 6,63 & 26,84 & 277,46 \\
\hline
\end{tabular}


M. S. ABDOU BABAYE et al. / Int. J. Biol. Chem. Sci. 10(6): 2728-2743, 2016

\begin{tabular}{|c|c|c|c|c|c|c|c|c|c|c|c|c|c|c|c|}
\hline Bogue II & F58 & G & 0,59832 & 14,04871 & 410 & 6,2 & 33 & 45,91 & 10,52 & 32,37 & 2,03 & 14,48 & 15,05 & 73,77 & 163,33 \\
\hline Ayoun Gam & F59 & G & 0,60216 & 14,25224 & 266,8 & 6,3 & 31,1 & 21,80 & 5,51 & 29,41 & 2,82 & 5,35 & 5,17 & 28,52 & 129,37 \\
\hline Sinessika & F61 & $\mathrm{G}$ & 0,88354 & 13,89807 & 539 & 6,9 & 32,2 & 36,78 & 20,14 & 63,39 & 2,92 & 10,28 & 17,44 & 16,82 & 306,72 \\
\hline SATOM & F24 & G & 0,731 & 14,01581 & 549,3 & 7,1 & 32,2 & 36,62 & 11,58 & 75,52 & 2,79 & 3,50 & 26,10 & 1,05 & 353,60 \\
\hline Begorou & F35 & G & 0,63666 & 14,03795 & 448 & 6,3 & 32,2 & 41,51 & 7,05 & 49,98 & 1,57 & 22,27 & 22,70 & 26,93 & 204,05 \\
\hline Tera Prison & $\mathrm{P} 2$ & Altérites & 0,74809 & 14,0066 & 719 & 7,1 & 32,9 & 74,78 & 27,38 & 33,62 & 6,26 & 42,10 & 21,75 & 138,24 & 211,25 \\
\hline
\end{tabular}

Tableau 2 : Matrice de corrélations sur les analyses globales des eaux souterraines.

\begin{tabular}{|c|c|c|c|c|c|c|c|c|c|c|c|c|}
\hline Paramètres & CE & pH & $\mathbf{C a}$ & Mg & $\mathbf{N a}$ & $\mathbf{K}$ & $\mathrm{Cl}$ & $\mathrm{SO}_{4}$ & $\mathrm{NO}_{3}$ & $\mathrm{HCO}_{3}$ & MT & $\mathrm{pCO}_{2}$ \\
\hline $\mathrm{CE}$ & 1 & & & & & & & & & & & \\
\hline $\mathrm{pH}$ & 0.12 & 1 & & & & & & & & & & \\
\hline $\mathrm{Ca}$ & 0.91 & 0.02 & 1 & & & & & & & & & \\
\hline $\mathrm{Mg}$ & 0.78 & 0.39 & 0.71 & 1 & & & & & & & & \\
\hline $\mathrm{Na}$ & 0.55 & 0.32 & 0.39 & 0.42 & 1 & & & & & & & \\
\hline $\mathrm{K}$ & 0.21 & 0.19 & 0.29 & 0.15 & 0.02 & 1 & & & & & & \\
\hline $\mathrm{Cl}$ & 0.80 & -0.22 & 0.84 & 0.50 & 0.37 & 0.11 & 1 & & & & & \\
\hline $\mathrm{SO}_{4}$ & 0.47 & 0.08 & 0.49 & 0.49 & 0.37 & 0.23 & 0.15 & 1 & & & & \\
\hline $\mathrm{NO}_{3}$ & 0.85 & -0.09 & 0.89 & 0.62 & 0.33 & 0.10 & 0.91 & 0.18 & 1 & & & \\
\hline $\mathrm{HCO}_{3}$ & 0.14 & 0.73 & -0.01 & 0.43 & 0.53 & 0.17 & -0.22 & 0.13 & -0.21 & 1 & & \\
\hline MT & 0.94 & 0.24 & 0.92 & 0.86 & 0.64 & 0.24 & 0.75 & 0.55 & 0.81 & 0.29 & 1 & \\
\hline $\mathrm{pCO}_{2}$ & -0.16 & -0.83 & -0.10 & -0.29 & -0.10 & -0.12 & 0.06 & 0.00 & -0.10 & -0.29 & -0.19 & 1 \\
\hline
\end{tabular}




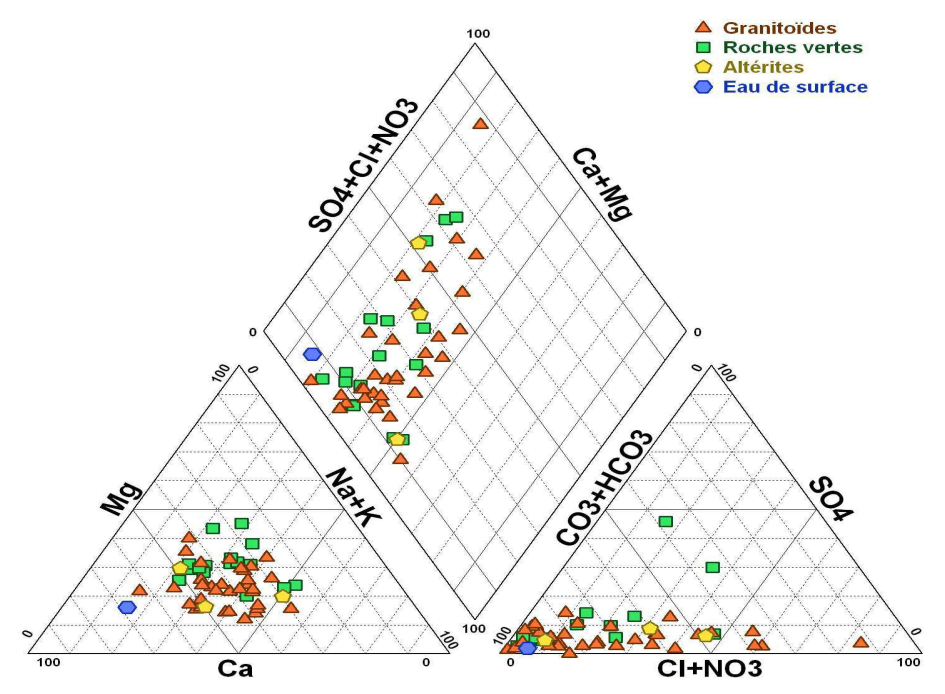

Figure 2 : Faciès chimiques de l'ensemble des eaux.
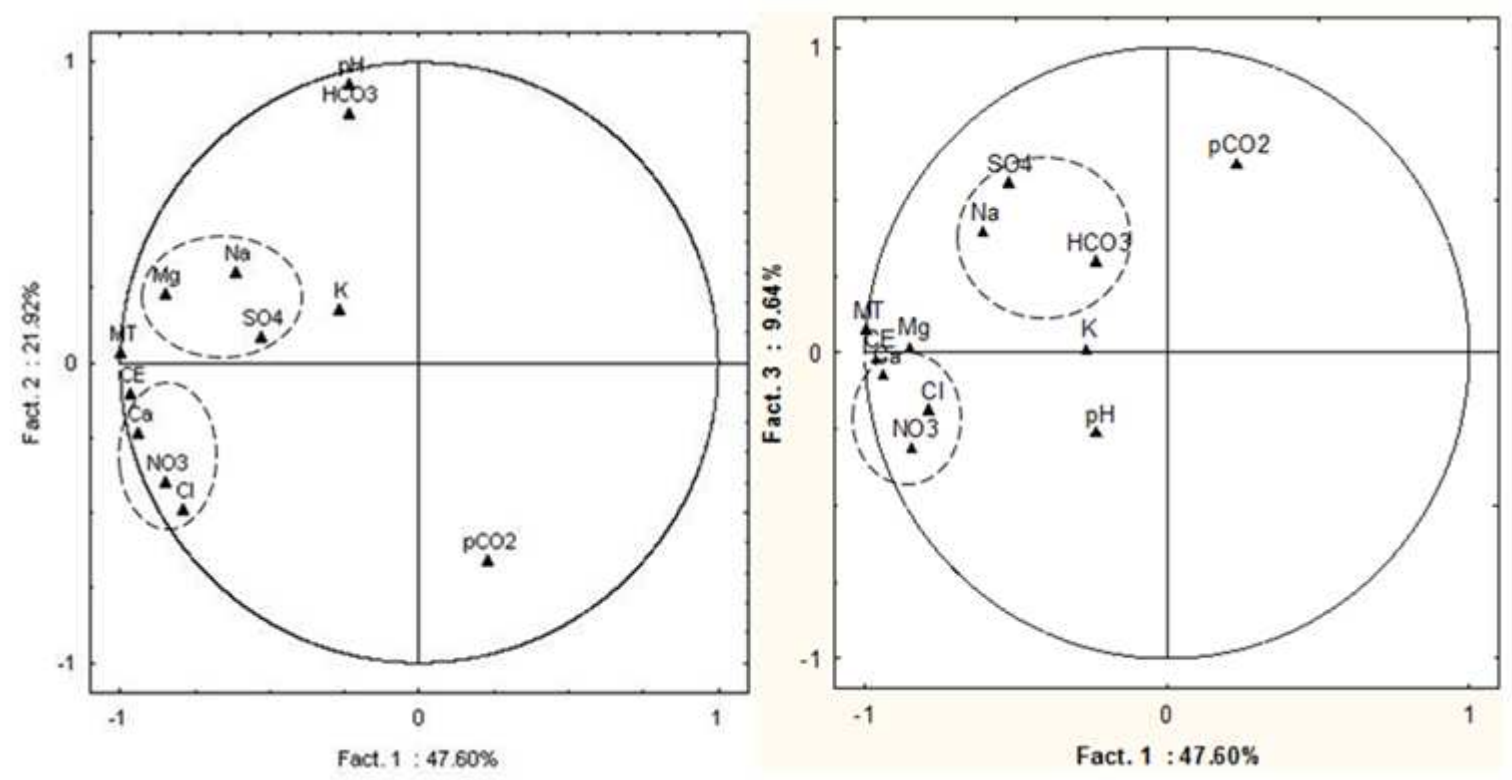

Figure 3 : Projection des variables sur les plans factoriels $F_{1}-F_{2}$ et $F_{1}-F_{3}$.

de la minéralisation de l'eau dans l'aquifère. $\mathrm{La}$ corrélation $\mathrm{Mg}^{2+}, \mathrm{Na}^{+}, \mathrm{SO}_{4}{ }^{2-}$ met en évidence la relation eau-roche, tandis que la présence des nitrates en excès dans le groupe $\mathrm{Ca}^{2+}-\mathrm{NO}_{3}{ }^{-}-\mathrm{Cl}^{-}$exprime la minéralisation rapide due aux activités anthropiques. Le facteur $\mathrm{F}_{2}$ explique près de $22 \%$ de l'inertie totale, discrimine la source principale de minéralisation et oppose la $\mathrm{pCO}_{2}$ au groupement $\mathrm{HCO}_{3}{ }^{-}-\mathrm{pH}$. La corrélation de l'ion $\mathrm{HCO}_{3}{ }^{-}$avec le $\mathrm{pH}$ montre que cet ion provient en majorité de l'hydrolyse acide des minéraux 
silicatés due à la présence de $\mathrm{CO}_{2}$ du sol. L'axe $\mathrm{F}_{3}$ contrôlant $10 \%$ d'informations, discrimine davantage les 2 sources de minéralisations déjà identifiées. Dans le plan factoriel $\mathrm{F}_{1}-\mathrm{F}_{3}$, l'opposition du groupement $\mathrm{Cl}^{-}$ - $\mathrm{NO}_{3}{ }^{-}-\mathrm{Ca}^{2+}$ à celui du $\mathrm{HCO}_{3}{ }^{-}-\mathrm{Na}^{+}-\mathrm{Mg}^{2+}$ confirme que la production des ions composant ces deux sous-groupes est dominée respectivement par la minéralisation superficielle et l'interaction eau - roche. La présence de l'ion $\mathrm{Ca}^{2+}$ entre ces deux sousgroupes montre qu'il pourrait avoir deux origines.

La projection des individus sur le plan factoriel $F_{1} \times F_{2}$ montre que le regroupement des échantillons se fait selon la minéralisation et le taux du $\mathrm{CO}_{2}$ contenu dans l'eau (Figure 4). Les eaux fortement minéralisées se placent sur le pôle négatif de l'axe $\mathrm{F}_{1}$ et celles ayant une minéralisation faible à l'opposé.

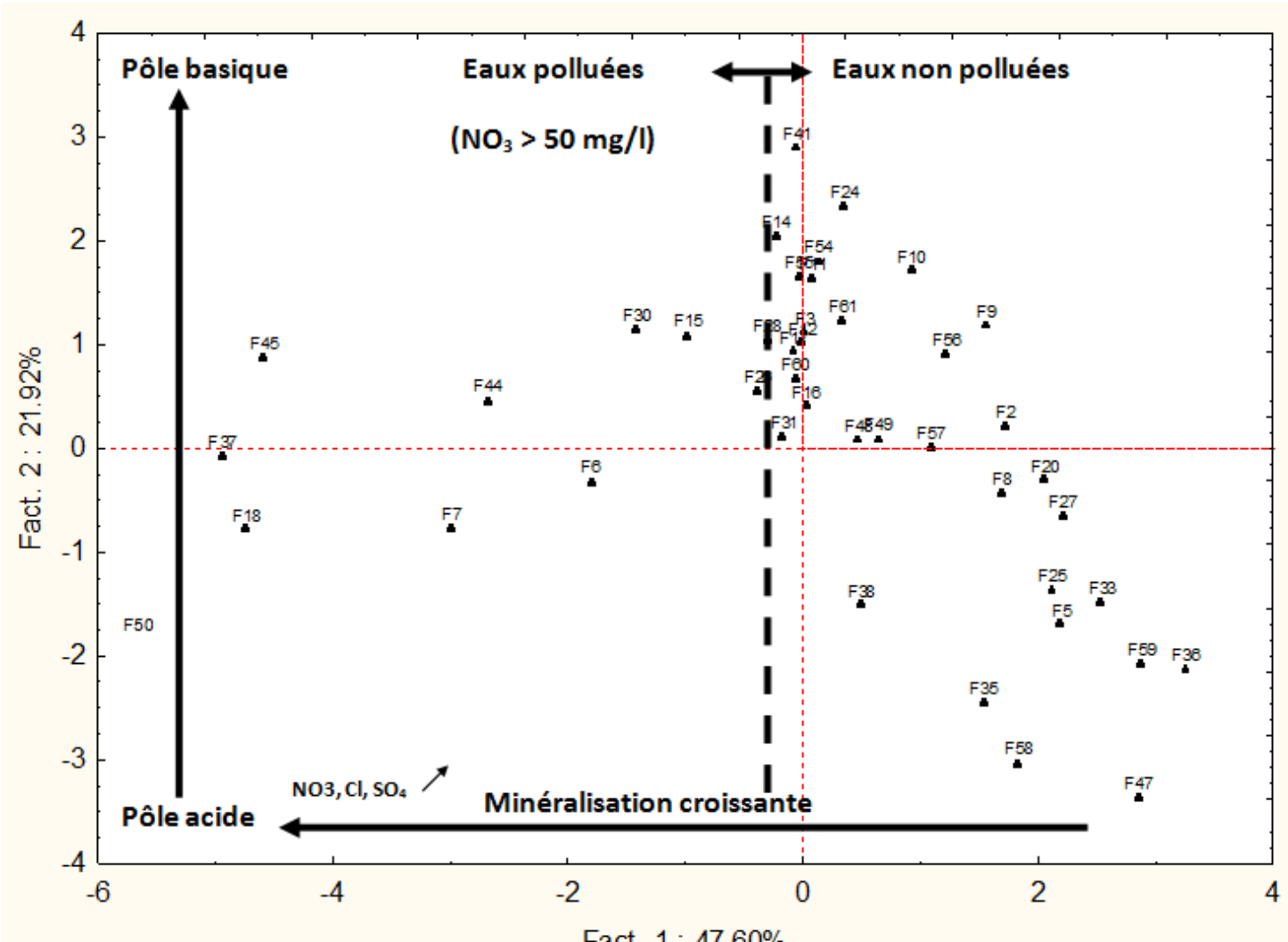

Figure 4 : Projection des individus sur le plan factoriel $F_{1} \times F_{2}$.

Tableau 3 : Valeurs propres et pourcentages des variances exprimées par les axes principaux.

\begin{tabular}{ccccc}
\hline Plans factoriels & $\begin{array}{c}\text { Valeurs } \\
\text { propres }\end{array}$ & $\begin{array}{c}\text { \% de variance } \\
\text { exprimée }\end{array}$ & $\begin{array}{c}\text { cumul de valeurs } \\
\text { propres }\end{array}$ & $\begin{array}{c}\text { \% de variance } \\
\text { exprimée cumulée }\end{array}$ \\
\hline F1 & 5,71 & 47,6 & 5,71 & 47,6 \\
\hline F2 & 2,63 & 21,92 & 8,34 & 69,52 \\
\hline F3 & 1,16 & 9,64 & 9,5 & 79,16 \\
\hline
\end{tabular}




\section{DISCUSSION}

\section{Contrôle hydrogéochimique des eaux}

Les eaux des aquifères profonds de socle montrent une diversité des faciès avec une dominance bicarbonaté calco-magnésien. En région de socle cristallin et cristallophyllien, le facteur le plus déterminant dans l'acquisition des cations est l'hydrolyse des minéraux silicatés. Le contact prolongé de l'eau chargée en $\mathrm{CO}_{2}$ avec l'encaissant se traduit par un enrichissement en cations majeurs $\left(\mathrm{Ca}^{2+}, \mathrm{Mg}^{2+}, \mathrm{Na}^{+}, \mathrm{K}^{+}\right)$et en ions bicarbonates. L'abondance de ces ions dans les eaux souterraines de socle de l'Afrique de l'Ouest est confirmée par plusieurs études (Oga, 2009 ; Lasm et al., 2011 ; Ahoussi et al., 2012 ; Abdou Babaye, 2012). Néanmoins, ces eaux enregistrent une faible teneur en potassium du fait de leur adsoption par les argiles de néoformation et les plantes (Oga, et al., 2009). Selon Abdou Babaye (2012), les eaux circulant dans les roches basiques seront plus riches en alcalino-terreux, alors que celles des roches acides sont à dominance alcaline (Figure 5a). À travers ce graphe, on perçoit clairement que la minéralisation des eaux est intimement liée à la nature pétrographique de l'encaissant (Adiaffi et al., 2009 ; Kouassi et al., 2012). Cela est aussi mieux discriminé par le rapport caractéristique $\mathrm{Mg} / \mathrm{Na}$. Plus de $90 \%$ des eaux de forages captant les roches acides ont un rapport $\mathrm{Mg} / \mathrm{Na}<1$ alors qu'il devient négatif pour $77 \%$ des eaux circulant dans les fissures des roches basiques. Certains forages $\left(F_{3}, F_{8}\right)$, bien étant sur le terrain schisteux, leur proximité avec la zone de recharge perturbe cet équilibre avec l'apport superficiel de $\mathrm{Na}^{+}$ (Lasm et al., 2011) dépassant largement le $\mathrm{Mg}^{2+}(\mathrm{Na}>\mathrm{Ca}>\mathrm{Mg}>\mathrm{K})$. Par contre, d'autres forages, bien qu'ils soient sur le terrain granitique, peuvent avoir des intrusions doléritiques riches en ferromagnésiens. L'utilisation de $\mathrm{HCO}_{3}{ }^{-}$comme indicateur du processus chimique dominant de production d'ions dans les nappes de socle, est à relier avec le mode de sa formation (Kouassi et al., 2011). Les bicarbonates sont produits suite à l'apport du $\mathrm{CO}_{2}$ par l'eau d'infiltration, qui par réaction d'hydrolyse des silicates permet, d'une part la formation du $\mathrm{HCO}_{3}^{-}$, et d'autre part, l'augmentation du $\mathrm{pH}$ en supposant une évolution en milieu fermé. On observe une corrélation linéaire entre les ions $\mathrm{Na}^{+}$et $\mathrm{Mg}^{2+}$ avec les bicarbonates (Figure 5b, 5c). Cette tendance à l'augmentation est moins significative avec le sodium. S'il est admis que l'ion $\mathrm{Mg}^{2+}$ provient en majorité de l'altération des minéraux silicatés ferromagnésiens (Ahoussi et al., 2012), une partie non négligeable de $\mathrm{Ca}^{2+}, \mathrm{Na}^{+}$et $\mathrm{K}^{+}$pourrait avoir une origine indépendante de l'hydrolyse des minéraux (Lasm et al., 2011). Cela est prouvé par l'ACP avec un regroupement des ions $\mathrm{Ca}^{2+}-\mathrm{NO}_{3}{ }^{-}-\mathrm{Cl}^{-}$opposé à celui de $\mathrm{Mg}^{2+}$ $\mathrm{Na}^{+}-\mathrm{SO}_{4}{ }^{2-}$. La présence de deux sous-groupes au niveau du $\mathrm{F}_{1}$ relate respectivement l'affinité des ions $\mathrm{Ca}^{2+}-\mathrm{NO}_{3}{ }^{-}-\mathrm{Cl}^{-}$et $\mathrm{Mg}^{2+}-\mathrm{Na}^{+}$$\mathrm{SO}_{4}{ }^{2-}$ et leurs actions dans la minéralisation de l'eau. Le premier sous-groupe exprime l'influence des activités anthropiques dans l'acquisition de la minéralisation des eaux qui s'explique en partie par la présence de l'ion $\mathrm{NO}_{3}^{-}$(Yaméogo, 2008; Adiafi, 2008; Ahoussi et al., 2012).

La Figure 5d discrimine les échantillons ayant une teneur appréciable de $\mathrm{Ca}^{2+}$ qui s'accompagne avec des teneurs importantes en $\mathrm{NO}_{3}^{-}, \mathrm{Cl}^{-}$et $\mathrm{SO}_{4}{ }^{2-}$. A l'image de toutes les zones arides, la zone d'étude est soumise à une évaporation importante due aux températures élevées pendant les longues saisons sèches. Ce phénomène va favoriser la formation et la concentration des certains sels minéraux comme le gypse $\left(\mathrm{CaSO}_{4}\right)$. Le dépôt de ces sels dans les zones des fractures et leur mise en solution par l'eau d'infiltration favorisent leur migration vers les nappes (Zuppi et al., 2004; Ahoussi et al., 2012). Selon Schoeller (1962), le processus de la dissolution des sels refermant les ions $\mathrm{Ca}^{2+}$ entraîne la diminution des ions $\mathrm{HCO}_{3}{ }^{-}$et $\mathrm{CO}_{3}^{-}$ 
dans les eaux. La figure 6a met en évidence ce phénomène et par conséquent, on admet qu'une partie des ions $\mathrm{Ca}^{2+}$ provient de la dissolution du gypse. S'agissant de l'origine des ions $\mathrm{NO}_{3}^{-}$et $\mathrm{Cl}^{-}$, leur forte corrélation prouve une même origine superficielle différente de celle des sulfates (Figure 6b). Les points à forte minéralisation se caractérisent par des teneurs élevées en $\mathrm{NO}_{3}^{-}$ et $\mathrm{Cl}^{-}$qui ne s'accompagnent pas avec celle des $\mathrm{SO}_{4}{ }^{2-}$. Cette différence exprimée sur les forages de Téra $\left(\mathrm{NO}_{3}^{-}=690,5 \mathrm{mg} / 1, \mathrm{Cl}^{-}=\right.$ $106,81 \mathrm{mg} / \mathrm{l})$ et de Kabangou $\left(\mathrm{NO}_{3}{ }^{-}=405,83\right.$ $\mathrm{mg} / \mathrm{l}, \mathrm{Cl}^{-}=45 \mathrm{mg} / \mathrm{l}$ ), qui ont des teneurs faibles en $\mathrm{SO}_{4}{ }^{2-}$, sont respectivement de 28,27 $\mathrm{mg} / \mathrm{l}$ et $15,42 \mathrm{mg} / \mathrm{l}$. L'isolement de certaines fractures favorisent le contact prolongé eauroche et limite l'arrivée d'eau venant d'autres fractures. Les hétérogénéités pétro-structurale et géomorphologique accentuent la forte variabilité des charges minérales des eaux souterraines de socle (Lasm et al., 2011; Ahoussi et al., 2012 ; Kouassi et al., 2012).

\section{Pollution des nappes}

Les activités anthropiques constituent un aspect important dans l'acquisition de la minéralisation des eaux. L'agriculture et l'élevage apportent divers éléments chimiques $\left(\mathrm{Na}^{+}, \mathrm{NO}_{3}^{-}, \mathrm{Cl}^{-}, \mathrm{SO}_{4}{ }^{2-}, \ldots\right)$ qui seront entraînés jusqu'à la nappe par les eaux d'infiltration (Schwiede et al., 2005 ; Ahoussi et al., 2012). L'accumulation de ces éléments dissous dans les nappes peut parfois dépasser les normes de potabilité et augmente de ce fait le risque des maladies d'origines hydriques. L'ACP réalisée sur les eaux souterraines (Figure 3) montre que l'ion $\mathrm{NO}_{3}^{-}$est d'origine superficielle. Si on admet l'hypothèse selon laquelle les nitrates sont d'origines superficielles, la nappe aurait donc sa teneur maximale en fin des saisons pluvieuses. La Figure 7 montre que la totalité des ouvrages atteint le seuil maximum en nitrates pendant la saison pluvieuse. Cette observation illustre bien l'apport de cet ion par les eaux d'infiltration récentes (Hamza et al., 2007 ; Lasm et al., 2011). On remarque aussi que les aquifères des roches basiques sont moins sensibles à la pollution que ceux des roches acides. Cette faible sensibilité est due à la nature géologique de leurs produits d'altération composés des matériaux à dominance argileuse, moins perméables que ceux des roches granitiques, mais aussi dû à la présence des matières organiques dans les argiles favorisant les activités bactériennes. L'importance des pollutions nitratées (40\% d'ouvrages) dans cette région aride sans activités agricoles de grande envergure impose la recherche des différentes sources de contamination. Les premiers indices indiquent que cet ion arrive dans la nappe par les eaux d'infiltration et que tous les forages contaminés se trouvent soit à proximité des mares ou dans le creux des dunes pour les zones rurales, soit proche des latrines ou d'égout pour le centre sémi-urbain de Téra. La zone d'étude est par essence une zone d'élevage où les animaux et les hommes sont alimentés par les eaux des forages (ou puits) pendant toute la période sèche ( 8 mois). Cette pollution est certainement liée au long séjour des excréments des animaux aux abords des points d'eau lors de leur abreuvage (Schwiede et al., 2005; Chibane et al., 2010). Cette observation est cohérente en ce sens que tous les points d'eau ruraux contaminés sont à proximités des villages d'éleveurs peulhs ou touaregs. Donc, la teneur anormalement élevée des nitrates dans les eaux souterraines est liée aux activités anthropiques accentuées par le caractère pastoral de la zone. On remarque bien que l'origine des nitrates en zone de socle de l'Afrique de l'Ouest est très diversifiée (Yaméogo, 2008; Ahoussi et al., 2012). Les travaux de Schwiede et al. (2005) ont prouvé la présence des teneurs élevées de nitrate sous les termitières et les arbres (acacia). La disparition de ces derniers suite à la déforestation aigue constatée ces dernières années pourrait être à l'origine de la 
concentration élevée des ions $\mathrm{NO}_{3}^{-}$dans les eaux souterraines. Ce fait est observé dans les pays sahéliens à travers les défrichages d'arbres pour les utilisations domestiques, mais aussi la baisse de la pluviométrie entraînant la disparition des certains gros arbres (Favreau, 2009) qui favorisera à son tour la drainance d'azote non fixé par les eaux d'infiltration vers la nappe. Ainsi, la pollution des forages profonds est due à l'héritage d'une forte activité biologique au moment de la recharge. En revanche, certaines études dans les villes Ouest africaines (Yaméogo, 2008 ;
Ahoussi et al., 2010) lient cette pollution aux activités anthropiques de la population entraînant la production des déchets domestiques. La proximité des ouvrages très pollués avec le réseau d'égout et des latrines montre l'effet de la contamination anthropique des eaux souterraines. La présence des teneurs importantes des nitrates rencontrées dans des nappes profondes de socle que proches de la surface, est due aux mécanismes d'écoulement préférentiel favorisant leur migration rapide et leur dispersion à travers des macropores dû aux fractures.
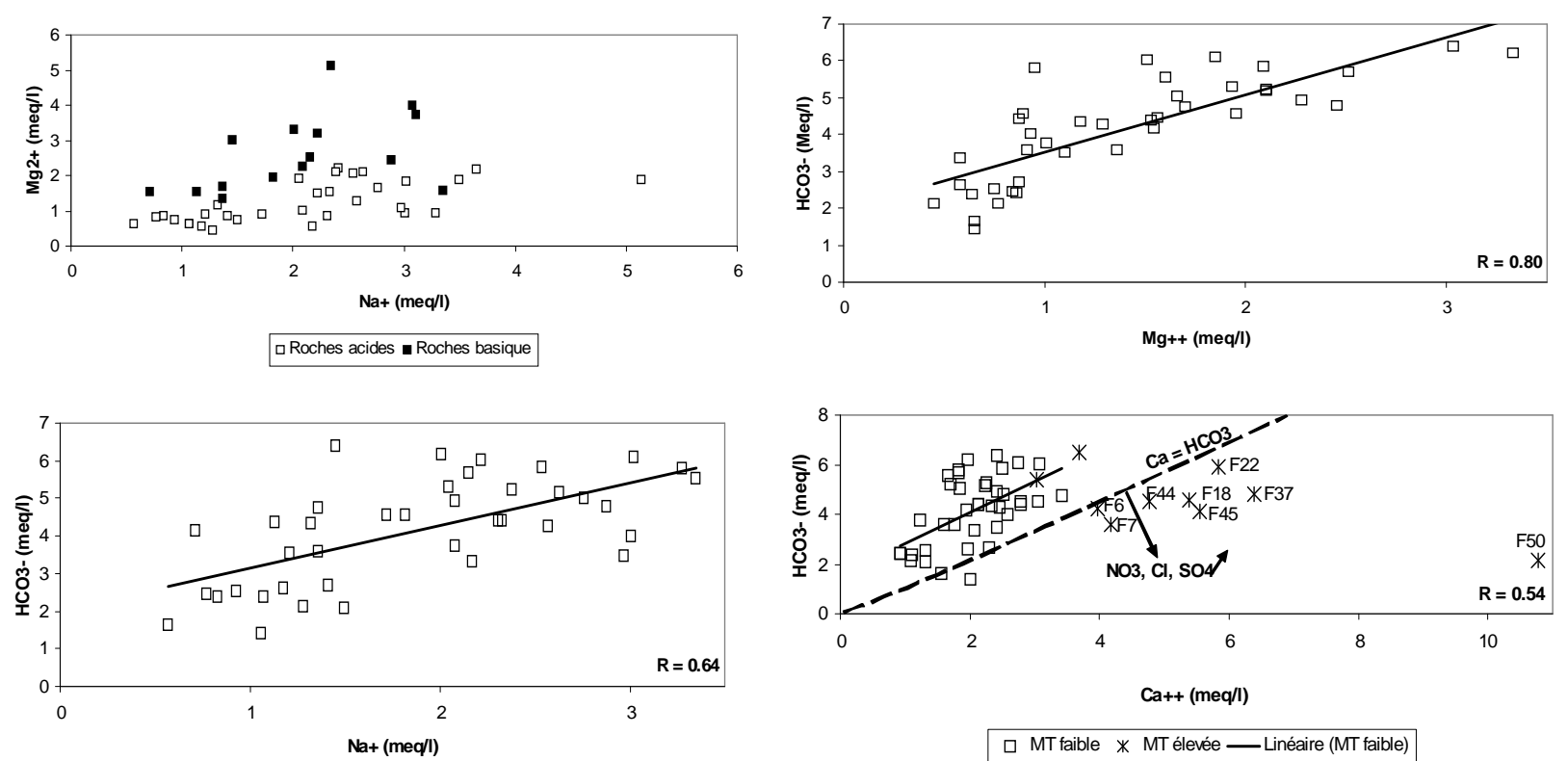

Figure 5 : Relations $\mathrm{Mg}^{2+}$ et $\mathrm{Na}^{+}(\mathrm{a})$ et cations avec les bicarbonates (b, c, d).

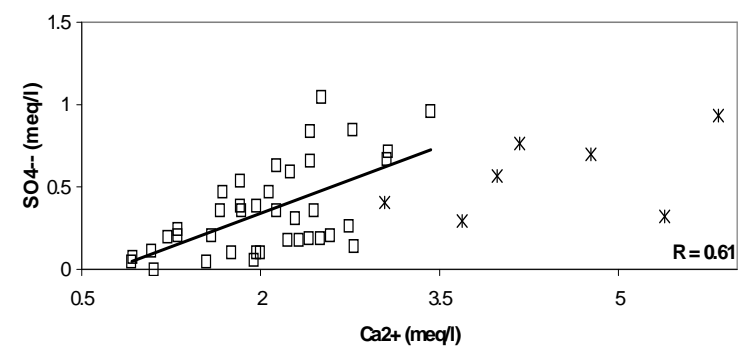

૫ MT faible $*$ MT élevée — Linéaire (MT faible)

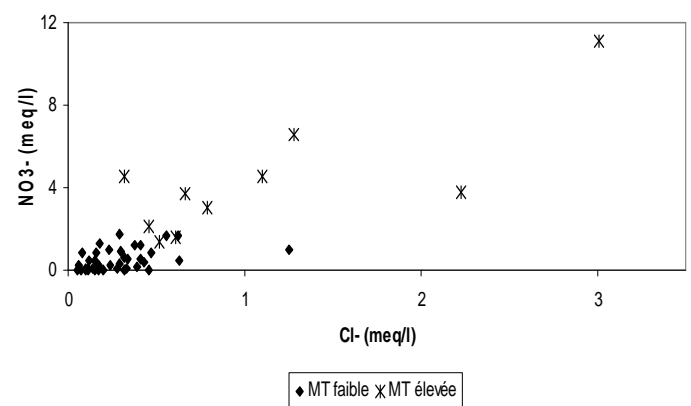

Figure 6 : Relation entre $\mathrm{Ca}^{2+}$ et $\mathrm{SO}_{4}{ }^{2-}$ (a) et $\mathrm{NO}_{3}{ }^{-}$avec $\mathrm{Cl}^{-}$(b). 


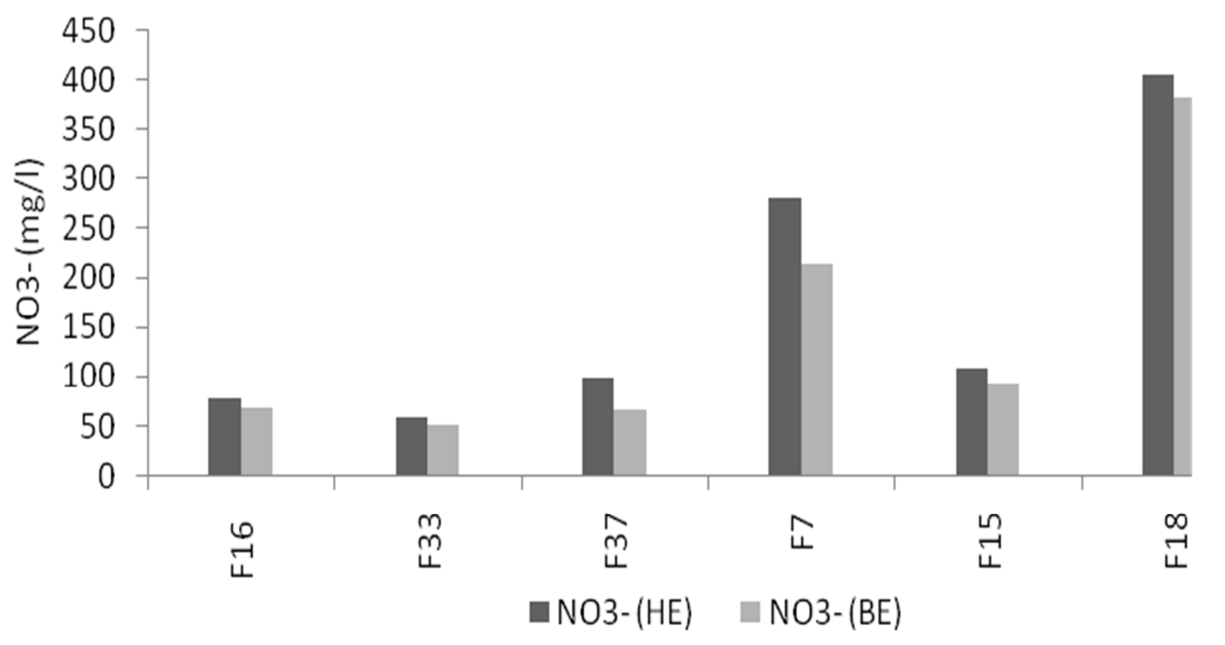

Figure 7 : variation de $\mathrm{NO}_{3}{ }^{-}$entre hautes eaux (HE) et basses eaux (BE).

\section{Conclusion}

L'étude hydrogéochimique des eaux souterraines a permis de caractériser les aquifères de socle. Les eaux de ces aquifères ont un $\mathrm{pH}$ acide avec une tendance vers la neutralité. Dans l'ensemble, ces eaux sont faiblement minéralisées. Cependant, on rencontre des eaux très chargées avec des valeurs de conductivité électrique pouvant atteindre $1731 \mu \mathrm{S} / \mathrm{cm}$. Cette hausse de la minéralisation est due à la contamination des eaux à des teneurs élevées de $\mathrm{NO}_{3}{ }^{-}$rendant celles-ci impropres à la consommation humaine. La classification chimique des eaux révèle une dominance des eaux bicarbonatées calco-magnésiennes (70\%), suivies des eaux chloruro-sulfatées calciques (25\%) et des eaux bicarbonatées sodiques (5\%). L'origine de la minéralisation dans les aquifères de socle, est en partie liée à la nature pétrographique et géochimique de l'encaissant, par l'action d'hydrolyse des minéraux silicatés, mais aussi aux apports superficiels. Les nitrates proviennent des pollutions anthropiques notamment les effluents d'égout ou des latrines dans le centre semi-urbaine, et des déjections animales dans les zones rurales. Les études isotopiques permettront de préciser à l'avenir ces hétérogénéités hydrochimiques, afin de comprendre leur rôle dans l'hydrodynamisme des nappes, mais aussi de bien discriminer les sources des ions $\mathrm{NO}_{3}{ }^{-}$et $\mathrm{SO}_{4}^{-2}$.

\section{CONFLIT D'INTERETS}

Les auteurs déclarent qu'ils n'ont aucun conflit d'intérêts.

\section{REMERCIEMENTS}

Cette étude a été réalisée avec l'aide logistique de l'Université Abdou Moumouni (UAM). Nous lui exprimons notre profonde gratitude.

\section{RÉFÉRENCES}

Abdou A, Bonnot H, Bory KD, Chalamet D, St Martin M, Younfa I. 1998. Notice explicative des cartes géologiques du Liptako à $1 / 100 \quad 000$ et $1 / 200 \quad 000$. Ministère des mines et de l'énergie du Niger, p.64.

Abdou Babaye MS. 2012. Evaluation des ressources en eau souterraine dans le bassin de Dargol (Liptako Niger). PhD Thesis, Université Liège, p235.

Adiaffi B. 2008. Apport de la géochimie isotopique, de l'hydrochimie et de la télédétection à la connaissance des 
aquifères de la zone de contact "soclebassin sédimentaire" du Sud-Est de la Côte d'Ivoire. Thèse de doctorat, Université Paris Sud, p.230.

Adiaffi B, Marlin C, Oga YMS, Massault M, Noret A, Biémi J. 2009. Palaeoclimatic and deforestation effect on the coastal fresh groundwater resources of SE Ivory Coast from isotopic and chemical evidence. J. Hydrol., 1-2: 130-141. doi:10.1016/j.jhydrol.2009.02.046.

Ahoussi KE, Soro N, Koffi BY, Soro G, Biemi J. 2010. Origine de la minéralisation des eaux des aquifères discontinus sous couvert forestier de la zone Sud de la Côte d'Ivoire : cas de la région d'AbidjanAgboville. Int. J. Biol. Chem. Sci., 4(3): 782-797.

DOI : http://ajol.info/index.php/ijbcs.

Ahoussi KE, Youan TM, Loko S, Adja MG, Lasm L, Jourda JP. (2012). Étude hydrogéochimique des eaux des aquifères de fractures du socle Paléoprotérozoïque du Nord-Est de la Côte d'Ivoire : Cas de la région de Bondoukou. Afriq. Sci., 08(3) : $51-68$.

http://www.afriquescience.info/document. php?id=2565.

Chibane B, Boutaleb A, Lacroix M. 2010. Etude hydrochimique et Approche isotopique en Région semi-aride: cas du Synclinal de Djelfa (Algérie). Eur. J. Sci. Res., $\quad$ 45(2): 270-290. http://www.eurojournals.com/ejsr.htm.

Dewandel B, Lachassagne P, Wyns R, Marechal JC, Krishnamurthy NS. 2006. A generalized 3-D geological and hydrogeological conceptual model of granite aquifers controlled by single or multiphase weathering. Journal of Hydrology, 330: 260-284. doi:10.1016/j.jhydrol.2006.03.026.

Elbaz-Poulichet F, Favreau G, Leduc C, Seidel JL. 2002. Major ion chemistry of ground waters in the Continental Terminal water table of southwestern Niger (Africa). Applied Geochemistry, 17: 1343-1349. PII: S0883-2927(02)00024-0.
Favreau G, Cappelaere B, Massuel S, Leblanc M, Boucher M, Boulain N, Leduc C. 2009. Land clearing, climate variability, and water resources increase in semiarid southwest Niger: A review. Water Resour. Res., $\quad 45$ : $1-18$. DOI:10.1029/2007WR006785.

Girard P. 1993. Techniques isotopiques $\left({ }^{15} \mathrm{~N}\right.$, ${ }^{18} 0$ ) appliquées à l'étude des Nappes des altérites et du socle fracturé de l'ouest africain. Étude de cas: l'Ouest du Niger. Thèse de doctorat Université Québec, Montréal, p141.

Hamza MH, Added A, France A, Rodriguez R. 2007. Validité de l'application des méthodes de vulnérabilité DRASTIC, SINTACS et SI à l'étude de la pollution par les nitrates dans la nappe phréatique de Metline-Ras Jebel-Raf Raf (Nord-Est tunisien). C. R. Geoscience, 339 : 493505. doi:10.1016/j.crte.2007.05.003.

Kouassi MA, Ahoussi EK, Koffi BY, Ake YA, Biemi J. 2012. Caractérisation hydrogéochimique des eaux des aquifères fissurés de la zone Guiglo-Duekoué (Ouest de la Côte d'Ivoire). Int. J. Biol. Chem. Sci., 6(1): 504-518. DOI : http://dx.doi.org/10.4314/ijbcs.v6i1.45.

Kouassi MA, Yao AK, Ake YA, Biemi J. 2011. Application des méthodes d'analyses statistiques multivariées à l'étude de la minéralisation des eaux des aquifères fissurés de la zone GuigloDuekoué (Ouest de la Côte d'Ivoire). Int. J. Biol. Chem. Sci., 5(5): 2155-2169. DOI : http://dx.doi.org/10.4314/ijbcs.v5i5.35

Leblanc M, Favreau G, Massuel S, Tweed S, Loireau M, Cappelaere B. 2008. Land clearance and hydrological change in the Sahel. SW Niger. Global and Planetary Change, 61(3-4): 135-150. doi:10.1016/j.gloplacha.2007.08.011.

Lasm T, De Lasme O, Oga MSY, Youan Ta M, Baka D, Kouame F, Yao TK. 2011. Caractérisation hydrochimique des aquifères fissurés de la région de SanPedro (Sud-Ouest de la Côte d'Ivoire). Int. 
J. Biol. Chem. Sci., 5(2): 642-662. http://ajol.info/index.php/ijbcs

Machens E. 1973. Contribution à l'étude des formations du socle cristallin et de la couverture sédimentaire de l'Ouest de la République du Niger. Mém. BRGM, (82) : p167.

Oga MS, Lasm T, Yao TK, Soro N, Saley MB, Kouassi D, Gnamba F. 2009. Caractérisation chimique des eaux des aquifères de fractures: Cas de la Région de Tiassalé en Côte D'Ivoire. European Journal of Scientific Research, 31(1): 7287. http://www.eurojournals.com/ejsr.htm

Ousmane B. 1988. Étude géochimique et isotopique des aquifères du socle de la bande sahélienne du Niger (Liptako, SudMaradi, Zinder-Est). Thèse de doctorat d'Etat, Université Niamey, p175.

Schoeller H. 1962. Les Eaux Souterraines: Hydrologie Dynamique et Chimique, Recherche, Exploitation et Évaluation des Ressources. Edi. Masson et $\mathrm{C}^{\mathrm{ie}}$. ; 642.

Schwiede M, Duijnisveld WHM, Bottcher J. 2005. Investigation of processes leading to nitrate enrichment in soils in the Kalahari Region, Botswana. Physics and Chemistry of the Earth, 30: 712-716. doi:10.1016/j.pce.2005.08.012.

Soumaila A. 2000. Étude structurale, pétrogaphique et géochimique de la ceinture de Diagorou-Darbani, Liptako, Niger Occidental (Afrique de l'Ouest). Thèse de doctorat, Université FrancheComté, p253.

Taylor C, Lambin E, Stephenne N, Harding R, Essery R. 2002). The influence of land use change on climate in the Sahel. J. Climate, 15: 3615-3629. DOI: http://dx.doi.org/10.1175/15200442(2002)015

Yameogo OS. 2008. Ressources en eau souterraine du centre urbain de Ouagadougou au Burkina Faso, qualité et vulnérabilité. Université Avignon et pays de Vaucluse, Université Ouagadougou, p245.

Zuppi GM, Jean-Paul NJP, Martial DM, Darmendrail X, Fudral S, Jusserand C, Nicoud G. 2004. Eaux fortement minéralisées et circulations profondes dans le socle. Exemple des Alpes francoitaliennes. C. R. Geoscience, 336 :13711378. doi:10.1016/j.crte.2004.08.004 